\title{
Lumen
}

Selected Proceedings from the Canadian Society for Eighteenth-Century Studies

\section{Bonheur et Vie matérielle d'après le Tableau de Paris de Louis-Sébastien Mercier}

\section{Hélène Cussac}

Volume 19, 2000

Material Productions \& Cultural Construction

Culture matérielle \& Constructions discursives

URI : https://id.erudit.org/iderudit/1012321ar

DOI : https://doi.org/10.7202/1012321ar

Aller au sommaire du numéro

Éditeur(s)

Canadian Society for Eighteenth-Century Studies / Société canadienne d'étude du dix-huitième siècle

ISSN

1209-3696 (imprimé)

1927-8284 (numérique)

Découvrir la revue

Citer cet article

Cussac, H. (2000). Bonheur et Vie matérielle d'après le Tableau de Paris de

Louis-Sébastien Mercier. Lumen, 19, 135-151. https://doi.org/10.7202/1012321ar

Copyright (c) Canadian Society for Eighteenth-Century Studies / Sociéte canadienne d'étude du dix-huitième siècle, 2000
Ce document est protégé par la loi sur le droit d'auteur. L'utilisation des services d'Érudit (y compris la reproduction) est assujettie à sa politique d'utilisation que vous pouvez consulter en ligne.

https://apropos.erudit.org/fr/usagers/politique-dutilisation/ 


\section{Bonheur et Vie matérielle d'après le Tableau de Paris de Louis-Sébastien Mercier.}

Je me sentis moins abattu après que j'eus mangé. C'est une chose admirable que la nourriture, lorsqu'on a du chagrin; il est certain qu'elle met du calme dans l'esprit; on ne saurait être bien triste pendant que l'estomac digère. ${ }^{1}$

Jacob s'en tient à la nourriture dans la tradition du valet soucieux de son plaisir de bouche. Au-delà de cette tradition comique, cette remarque renvoie au nouvel état d'esprit propre au XVIIIè siècle qui ne sépare plus plaisirs d'origine matérielle et impressions de bonheur. N'est-ce-pas une des leçons que le Tableau de Paris offre à son lecteur? Le quotidien s'y déploie en effet largement au-delà de la nourriture.

De 1781 à 1789, Louis-Sébastien Mercier nous ouvre, avec son Tableau de Paris, ${ }^{2}$ une mine $\mathrm{d}^{\prime}$ or $^{3}$ ethnologique, un ouvrage 'feuillu' au genre peut-être à jamais indéfinissable, mais grâce aux abondantes descriptions contenues dans ses douze tomes, le lecteur s'aperçoit qu'il a été, bien plus que les écrivains qui l'ont précédé et inspiré, attentif aux aspects matériels de la vie quotidienne. ${ }^{4}$ Si l'inspiration de son Tableau est un lointain héritage de Germain Brice, ${ }^{5}$ sa démarche se rapproche plutôt de celle de Poullain de Saint-Foix. ${ }^{6}$ Chez Mercier se lit un désir d'échapper aux contraintes de la pratique habituelle; aussi a-t-il un goût prononcé pour un parcours 'sans bornes,' ni esthétiques ni sociologiques:

J'ai fait des recherches dans toutes les classes de citoyens, et n'ai pas dédaigné les objets les plus éloignés de l'orgueilleuse opulence, afin de mieux établir par ces oppositions la physionomie morale de cette gigantesque capitale. (I, 13)

Paris apparaît tout d'abord comme un ensemble de désignations rigides propre à toute onomastique établie. ${ }^{7}$ La ville s'impose donc par le biais des noms et Mercier l'aborde avant tout avec un savoir quasi encyclopédique. En effet la multiplicité toponymique détermine clairement le projet d'établir un dictionnaire de la ville qui en serait comme le miroir. Elle reflète une compréhension de la totalité de l'espace urbain. Une des 
conséquences d'un tel projet est l'impossibilité du regard de se limiter ${ }^{8}$ à des lieux architecturaux, centralisés et officialisés ${ }^{9}$. De fait, Mercier, flâneur, 'voyeur' de multiples lieux, semble avoir été happé par un tourbillon de choses multiples:

En mettant la tête à la fenêtre, on considère l'homme qui fait des souliers pour avoir du pain, et l'homme qui fait un habit pour avoir des souliers, et l'homme qui ayant des habits et des souliers, se tourmente encore pour avoir de quoi acheter un tableau. On voit le boulanger et l'apothicaire, l'accoucheur et celui qui enterre, le forgeron et le joaillier, qui travaillent pour aller successivement chez le boulanger, l'apothicaire, l'accoucheur et le marchand de vin. (I, 28)

L'image qui nous est renvoyée est bien celle d'un monde qui se laisse découvrir par une vision attentive à maints détails mettant en place non seulement une sémiologie nouvelle et moderne ${ }^{10}$ de l'espace, mais surtout une morale de l'objet: les relations humaines passent par les produits fabriqués. L'objet est donc une médiation créatrice d'un espace humain. De site géographique, la ville, modelée par mille choses, se transforme en fonction des événements de l'histoire des hommes; elle devient alors un réseau de signes qu'il s'agit de déchiffrer à travers les objets qui jalonnent le parcours de Mercier et qui sont source d'un pathos dysphorique ou euphorique selon qu'ils favorisent ou diminuent le bonheur des hommes.

En quoi ces objets du Paris mercérien sont-ils censés participer au bonheur? Y aurait-il chez l'écrivain un dévoiement de la réflexion morale de l'âme vers le corps en raison de cette nouvelle attention à la dimension matérielle de la vie humaine?

\section{1 - Place et statut de la culture matérielle chez Mercier}

a) Vers une morale de l'objet:

Mercier ne semble pas s'engager sans équivoque dans la voie que laisserait supposer son esthétique libérée des préjugés classiques relatifs aux objets bas de la vie terrestre (corps et objets matériels ). Laisse-t-il même une place au bonheur matériel? Le doute pourrait s'imposer quand on se rappelle les définitions que Mercier donnait du bonheur:

Il est de la nature des êtres intelligents de se dématérialiser le plus possible afin de vivre du bonheur pur et plein, je veux dire du bonheur du sentiment. ${ }^{11}$ 
Le bonheur serait lié à la vie intérieure et en ce sens Mercier retrouverait le dualisme de la culture classique portée à repérer les trahisons de la morale par le corps. Il y a en effet du La Bruyère chez l'écrivain:

L'extrême urbanité est le résultat d'une infinité de points délicats qu'il faut saisir; elle n'existe réellement que chez certains hommes dont le caractère est élevé et l'âme très sensible. L'homme de cour possède parfaitement cette noble urbanité quoiqu'il ne l'ait pas dans le cour; c'est qu'il sent avec finesse et qu'il est attentif aux convenances. $(\mathrm{I}, 613)$

Toutefois, la conception mercérienne du bonheur est marquée par une nuance sensualiste qu'il avait exprimée plus nettement en 1763 à propos de l'homme de lettres:

Il trouve des charmes variés où les autres n'aperçoivent qu'une couleur triste et uniforme. Il n'a pas besoin de recourir à des objets étrangers; il n'a qu'à descendre en lui-même fouiller cette mine riche et profonde qui recèle des trésors inconnus. Son âme est ... heureuse par le sentiment qu'elle a de connaître, d'embrasser divers rapports et de jouir d'une foule de tableaux. ${ }^{12}$

Cette réflexion de Mercier signale que le monde intérieur où se manifeste le bonheur n'est pas coupé du monde extérieur; le bonheur sentimental résulte de la perception des objets matériels. Ce moraliste s'appuie sur des argumentations 'physiciennes'; il reconnaît à la suite de Voltaire que 'le défaut d'occupation' ou 'mollesse du corps ... détériore les facultés de l'âme' (I, 633). Dans le domaine esthétique, la position de Mercier serait davantage intransigeante. Ne s'est-il pas sarcastiquement moqué en effet du goût contemporain pour le joli qu'il attache aux gens de la bonne compagnie dont l'âme stérile aurait besoin d'artifices matériels pour éprouver la moindre émotion: 'L'agrément est le dernier trait que l'on puisse donner aux belles choses' (I, 635)? Privée de ce supplément ornemental, l'âme contemporaine ne ressentirait plus la beauté que comme 'une image intellectuelle faite pour la rêverie des philosophes ... une harmonie très souvent froide et dénuée de grâce' (I, 639-40). Il y aurait donc au moins un bon et un mauvais usage des objets matériels: Mercier ferait-il dépendre leur statut de leur capacité à produire du bonheur ou du malheur pour l'être humain aussi bien dans le domaine des sens que dans le domaine du cour? En dépit de ses hésitations de moraliste, le statut fondamental de l'objet ne relève-t-il pas chez lui de son besoin avoué de jouir de divers tableaux, lesquels n'offriraient pas un simple attrait pittoresque mais enrichiraient son âme sensible, ce qui expliquerait ainsi sa quête incessante de figures humaines mais aussi de détails, d'éléments familiers de la vie quotidienne. 


\section{b) En quête de l'objet:}

La capitale, restituée dans son espace socio-topographique, devient une carte énergétique où Mercier, aventurier du quotidien, effectue un va-etvient, une sorte de danse au milieu de ces 'objets si fantasques, si inconnus' (I, 1249) qui le fascinent. ${ }^{13}$ A contrario d'une relation de voyage, c'est à partir d'un regard sensible sur les objets de la rue qu'il interroge l'environnement de l'homme; il n'y a ainsi pas de séparation entre la littérature et la vie quotidienne, fut-elle grossière: 'Le voyez-vous cet homme qui, à l'aide de son croc, ramasse ce qu'il trouve dans la fange, et le jette dans sa hotte?' (I, 452). A travers un art du détail, la plume cerne et retranscrit le mouvement de la vie qui donne chair à des personnages jusqu'alors occultés par la tradition littéraire: ${ }^{14}$

Les boutiques de pâtissiers, de charcutiers, de rôtisseurs, frappent la vue dans tous les carrefours. L'enseigne est la chose même; on voit des langues fourrées, des jambons couronnés de laurier, de grasses poulardes, des pâtés vermeils, des gâteaux tout sucrés qui sont sur le devant: on dirait qu'il n'y a qu'à y porter la main. (I, 1010)

En outre, l'écrivain brosse le portrait d'une ville en action en faisant voir, comprendre les gestes du quotidien, restituant 'la chorégraphie des pratiques sociales. ${ }^{15} \mathrm{Et}$, tout en restant par ailleurs fidèle à ses origines esthétiques et intellectuelles (Voltaire, Rousseau, Diderot), il établit la relation entre l'espace, l'objet de cet espace et la constitution de la vie et de la pensée ${ }^{16}$ en nous soumettant une histoire en noir et blanc de chaque jour, de chaque lieu, de chaque objet, dans une esthétique entre ombre et lumière.

N'est-ce-pas en marchant sur des 'ponts tremblants' (I, 110), en traversant un 'ruisseau fangeux' (ibid.), que Mercier porte son regard sur des points de détails pour ainsi dire inattendus, et qui l'amènent à une interrogation quelque peu singulière pour celui qui désigne constamment Paris de 'superbe': 'pourquoi n'avoir pas des trottoirs, comme à Londres?' (I,111). Loin de s'arrêter uniquement à des sujets relevant d'un critère du commun, il s'oriente vers un espace structuré par des objets en apparence insolites, et pourtant signes de l'appréhension et de la compréhension urbaines.

Au hasard des ponts, des quais, des places publiques; de la Halle au Faubourg Saint-Honoré et au Pont-Neuf jusqu'à ces zones urbaines et périphériques que sont Chaillot, Passy, Petit-Gentilly, La Courtille, les Porcherons, Mercier explore un large territoire qui le conduit à constater par exemple qu'il n'y a plus de lanternes depuis seize ans. Des réverbères ont pris leur place' $(I, 175)$. Il se préoccupe de choses diverses telles que les enseignes (I, 177), les cheminées (I, 71), les écriteaux des rues (I, 
402), du fumier (I, 475), des fleurs et des fruits de la Halle (I, 178), des fosses d'aisance (I, 116), du pain (I, 27), du vin falsifié et de l'eau de vie $(\mathrm{I}, 219,268)$, de la vaisselle $(\mathrm{I}, 56)$. Il ne néglige pas les objets de brocante: bijoux, tableaux, estampes antiques, vases, bronzes, médailles (I, 808). Peut-être même est-il surpris devant 'des charrettes surchargées de meubles' (I, 1160)? devant 'tel fauteuil délabré [que] l'on promène'? devant 'la chaise percée qui voyage' (ibid.)? La découverte de l'objet-rue qu'il saisit est encore celle ' $d$ ' une femme qui porte sur son dos et sur sa tête une quarantaine de paires de culottes' (II, 144) et de 'souliers neufs [qu'un] malheureux avait cachés dans un torchon' (ibid.). Une autre fois, il s'intéresse aux 'fantaisies changeantes' de la mode: coiffures, vêtements, petits chiens, boucles de souliers, bijoux et tabatières (I, 415-20).

Enfin, dans sa perception très inhabituelle de l'espace, ${ }^{17}$ l'écrivain constate que:

La valeur intrinsèque des objets apparaît là dans son évidence philosophique; et $\mathrm{d}$ 'après leur utilité, les chemises, les matelas, les chaises, les redingotes, etc. trouvent beaucoup plus de partisans que les diamants, les bijoux, les livres, etc. (I, 1248)

Le Paris du siècle des Lumières se donne ainsi à voir dans une perspective inaccoutumée. Au gré des lieux traversés, la prospection n'impose pas la vision unique et figée d'un espace pour voyageurs où entrerait seulement une architecture d'apparat destinée à chanter la gloire du roi, mais une composition d'éléments petits, variés qui touchent à l'essentiel: comment panser les plaies de l'homme puisque sa vie doit s'accompagner du principe de plaisir? Ce bric-à-brac des rues parisiennes qui défie les habitudes rationnelles de l'esprit classique et bouscule l'ordre esthétique des Belles-Lettres s'intègre parfaitement bien dans la conscience du promeneur-philosophe hédoniste. Ou du moins s'intégrerait bien si trop souvent les objets de la vie matérielle ne se transformaient en obstacles malfaisants.

\section{2 - Les objets agressifs}

Repaire de 'tous les vices et de tous les maux entassés les uns sur les autres' (I, 122), c'est un organisme fragile, voire apocalyptique que Mercier met à nu. Les agressions physiques, déjà prononcées chez un Boileau $^{18}$ (Satire VI), à la suite d'un Juvénal, sont nombreuses: 'Les environs des marchés sont impraticables; les emplacements sont petits, resserés; et les voitures menacent de vous écraser' $(\mathrm{I}, 181) \cdot{ }^{19}$ Les dangers de circulation relèvent malheureusement bien souvent du 'fait divers': 
'Rien de si commun que la soudaine rupture des soupentes ou des roues. Vous avez le nez cassé ou une contusion au bras' (I, 133); aussi est-ce la raison pour laquelle l'écrivain en fait une constante du Tableau. Il dresse alors un véritable réquisitoire contre le riche: 'J'ai donc un peu le droit d'accuser le luxe barbare des voitures' $(\mathrm{I}, 108)$ et contre une mauvaise gestion de la cité:

Quel contraste choquant entre la magnifique rive droite du fleuve, et la rive gauche qui n'est point pavée et est toujours remplie de boue et d'immondices! Elle n'est couverte que de chantiers et de masures habitées par la lie du peuple. $(\mathrm{I}, 141)$

Mercier montre que les agressions physiques se doublent d'agressivité esthétique:

Des rues étroites et mal percées, des maisons trop hautes et qui interrompent la libre circulation de l'air, des boucheries, des poissonneries, des égouts, des cimetières, font que l'atmosphère se corrompt .... Les maisons d'une hauteur démesurée sont cause que les habitants du rez-de-chaussée et du premier étage sont encore dans une espèce d'obscurité, lorsque le soleil est au plus au point de son élévation. Les maisons élevées sur les ponts, outre l'aspect hideux qu'elles présentent, empêchent le courant d'air de traverser la ville (I, 114).

L'écrivain n'hésite pas à fouiner, humer, croquer des quartiers et dire sans mentir une vie difficile car '... les maisons sont puantes, et les habitants perpétuellement incommodés. Chacun a dans sa maison des magasins de corruption; il s'exhale une vapeur infecte de cette multitude de fosses d'aisance' (I, 116). C'est un point crucial que ces fosses d'aisance ${ }^{20}$ non seulement à cause des mauvaises odeurs et de l'air vicié qui s'en dégagent, mais aussi à cause de la nourriture que peut avaler le Parisien: un 'aliment imprégné de ces parties méphitiques et malfaisantes [les matières fécales]' (I, 116), d'autant plus que 'les cadavres que volent ou qu'achètent les jeunes chirurgiens pour s'exercer dans l'anatomie, sont souvent coupés en morceaux, et jetés dans les fosses d'aisance' (I, 117). ${ }^{21}$ Fosses, puits, égouts, ruisseaux d'urine, monceaux d'excréments $^{22}$ renvoient à une perception olfactive et visuelle fortement négative et nous transportent vers les bas-fonds que découvre Mercier à travers ces motifs hygiéniques. C'est dans ces zones de la 'populace' qu'il souligne les dangers qu'encourent les hommes en buvant du vin falsifié et de l'eau de vie: ${ }^{23}$ bagarres, dépense de l'argent prévu pour le foyer, santé précaire; et de surcroît: 
pendant ce temps les enfants au logis crient après la nourrice qui leur manque, pleurent sous les flèches aigües du froid qui gèlent leurs petites mains. Le père abruti est sourd à leur voix, emporte les meubles pièce à pièce, et les vend pour se replonger dans l'ivresse. $(\mathrm{I}, 269)$

Mais les hommes ont-ils l'occasion d'avoir une vie plus saine quand même l'eau est indice d'épidémies? 'Quand la rivière est trouble, on boit l'eau trouble' (I, 134) et le seul recours est la rivière car 'les fontaines publiques sont rares et mal entretenues' (ibid.). Paris est bien ce lieu de crainte qui alarme Mercier:

Quand on songe qu'il y a à Paris près d'un million d'hommes entassés sur le même point, ... il y a vraiment de quoi frémir sur la future subsistance de ce peuple; .... Il est bien sûr que chaque Parisien n'aura désormais du pain, que tant qu'on voudra bien permettre aux boulangers d'avoir de la farine. (I, 73)

A une période où l'afflux des provinciaux est massif, les problèmes de subsistance s'accroissent. Aussi l'écrivain se révolte-t-il en percevant les inégalités notoires et dénonce-t-il les 'richesses monstrueuses, le luxe scandaleux' (I, 12). 'Ils [les riches] aiment mieux nourrir des chevaux que des hommes,' dit-il en constatant que cet égoïsme des grands est certainement cause que 'la magnificence de la nation est toute à l'intérieur des maisons' (I, 228). ${ }^{24} \mathrm{Il}$ s'élève en outre contre la frivolité de la mode parisienne, ${ }^{25}$ douce tentation des riches qui se rendent ridicules, entraînant avec eux la nation entière:

la rage de la frisure a gagné tous les états. Garçons de boutiques, clercs de procureurs et de notaires, domestiques, cuisiniers, marmitons, tous versent à grands flots de la poudre sur leurs têtes, tous y ajustent des toupets pointus, des boucles étagées. (I, 94)

Et bien qu'admirant l'habileté des marchandes de modes, il n'hésite pas à se moquer de 'la fameuse poupée, le mannequin précieux, affublé des modes les plus nouvelles, enfin le "prototype inspirateur"' (I, 410). $S^{\prime}$ ajoutent à ces signes révélateurs du malaise urbain des agressions sonores. Les rues apparaissent en effet souvent envahies par un vacarme tonitruant: ${ }^{26}$ 'Le bruit, le tumulte est si considérable [dans les marchés], qu'il faut une voix plus qu'humaine pour se faire entendre' (I, 181). La pollution sonore est permanente, due avant tout au bruit des carrosses sur les rues pavées, qui 'ébranle les maisons' (I, 875) et abasourdit le passant: 
A cinq et un quart, c'est un tapage affreux, infernal. Toutes les rues sont embarrassées, toutes les voitures roulent en tous sens, volent aux différents spectacles, ou se rendent aux promenades. $(I, 874)^{27}$

Au fracas effroyable des carrosses, s'additionne le bruit des outils des artisans: 'Le marteau du forgeron et du maréchal-ferrant trouble quelquefois le sommeil du matin ...' $(\mathrm{I}, 879){ }^{28}$

Suite à ces observations critiques contre ce qui empêche le citadin de vivre heureux, l'aventure du quotidien joue-t-elle sur le seul autel de la corruption et des dangers de la rue? Comme le dit Henri Lafon: 'de même qu'il y a un nécessaire pour survivre, fait d'argent, de nourriture, il y a un nécessaire du plaisir, ${ }^{29}$ aussi, malgré la misère des conditions sociales, c'est toujours dans le sentiment que l'inventivité, l'ingéniosité, l'habileté manuelle sont pour Mercier les signes prometteurs du bonheur humain. On pourrait penser, par exemple, que les artisans ajoutent le vacarme de leurs activités professionnelles à la pollution sonore de la ville. Mercier souligne en effet la 'lime mordante' des artisans, 'le chaudronnier qui bat sa marmitte,' 'le charron qui cercle la roue' (I, 879-80), mais au lieu d'inclure ces remarques dans la liste des dysfonctionnements urbains, il en fait davantage une critique des 'paresseux qui sont encore au lit' (ibid.) pour glorifier le travail humain. Il ne faut donc pas lire Mercier dans le droit fil de la tradition satirique qui honnissait la ville par souci de sauver l'âme par le dénuement. Ce n'est pas l'objet en lui-même qui fait le malheur de l'homme comme le prétendent tous les moralistes, mais son mauvais usage. Peut-on aller plus loin en pensant que l'objet quotidien donne naissance et accompagne le bonheur de vivre en ville?

\section{3 - Vers une morale du bonheur matériel}

a) Les objets de charme et de plaisir:

Pour intégrer à cette recherche du bonheur le disparate qui donne souvent au Tableau de Paris 'une couleur triste et dominante' (I, 19), Mercier s'efforce de repérer toutes les beautés qui dans l'espace urbain peuvent émerveiller le regard ou enchanter l'imagination. Tout d'abord il lui arrive de se détendre plutôt que de se crisper au contact d'un spectacle désolant:

C'est un spectacle curieux que de voir tout à son aise, du haut d'un balcon, le nombre et la diversité des voitures qui se croisent et s'arrêtent mutuellement; les piétons qui, semblables à des oiseaux effrayés sous le fusil du chasseur, se glissent à travers les roues de tous ces chars prêts à les écraser. L'un qui franchit le ruisseau de peur de s'éclabousser, et qui, manquant l'équilibre, se couvre de 
boue des pieds à la tête; l'autre, qui pirouette en sens contraire, une face dépoudrée, et le parasol sous le bras. (I, 916)

L'écrivain ne se retient pas de sourire; il est vrai qu'il profite de sa position extérieure et supérieure qui favorise une attitude de spectateur de comédie; il est vrai qu'il se moque aussi discrètement de ces Parisiens à face poudrée qu'il n'aime pas; néanmoins, il saisit avec plaisir l'occasion qui lui permet de retourner l'agression première en divertissement. Ainsi glane-t-il les petits faits de la banalité avec humour:

rien n'est perdu à Paris, ainsi que dans le système éternel de la nature. L'atome, la chemise usée, la culotte trouée et le soulier déformé ne périssent point encore; rien ne s'anéantit; non, rien; il se trouve toujours des individus qui entrent avec justesse dans ces moules tout prêts. Ces culottes suspendues invitent les passants, et la tentation est égale au besoin. (I, 1207)

Sur son chemin, Mercier rencontre des objets qu'il transforme en mots d'un quotidien charmant; il en cueille une gerbe embaumée comme fleurs de printemps, comme fleurs de beautés; en sage voluptueux, il convoque les Divinités du plaisir: ${ }^{30}$

Un coup d'œil unique est celui que présentent au point du jour la halle aux fleurs et la halle aux fruits dans le printemps et l'été. On est surpris, enchanté; ... Flore et Pomone se donnant la main, n'ont jamais eu de plus beau temple. (I, 178)

Puis il transforme ces détails de la vie quotidienne en choses belles à voir, en bribes de mots beaux à entendre:

Tous les sens sont interrogés à chaque instant; on brise, on lime, on polit, on façonne. L'esprit peut-il demeurer immobile et froid, tandis que, passant devant chaque boutique, il est stimulé, éveillé de sa léthargie par le cri de l'art qui modifie la nature? (I, 25)

Mercier, nourri pourtant de l'Encyclopédie, semble avoir mis davantage l'accent sur les arts, qui, autant et peut-être plus que les idées fondatrices des Lumières, peignent une 'superbe capitale':

Que de substances se fondent dans un si petit espace! Paris peut être considéré comme un large creuset, où les viandes, les fruits, les huiles, les vins, la cannelle, le sucre, le café, les productions les plus lointaines viennent se mélanger. (I, 24)

Comme l'exprime Daniel Roche, 'avec le Tableau de Paris, on passe d'une économie du salut, de la rareté, à une économie visant au bonheur 
terrestre, à l'abondance relative, à l'utilité.'31 A Paris, en effet, 'Tout abonde; ... le poisson délicieux, l'huître verte, le faisan, le chapon et l'ananas, .... Il est des ouvriers de sensualité qui décorent la coupe de la volupté, et qui savent raffiner des plaisirs déjà exquis $(\mathrm{I}, 46) .{ }^{32}$ Mercier n'est pas pour autant un promeneur solitaire et rêveur, il sait s'arrêter pour écouter battre l'horloge du progrès. Pêle-mêle, tous 'les arts se perfectionnent' (I, 243): cuisine, musique, médecine, vêtements, poésie, littérature. Ainsi l'univers change: 'on s'occupe plus que jamais du soin de remédier aux abus' (I, 121). Les progrès sociaux tels que la sûreté nouvelle pendant les nuits (I, 164), l'éclairage des rues (I, 175), la subsistance et les lits que trouvent les victimes de la société (I, 120), participent au désir de l'auteur à retrouver sens et valeur que des siècles de barbarie avaient fait perdre au plaisir de vivre dans la capitale. Le trottoir dont parle souvent Mercier n'est pas un simple élément technique; il est un élément matériel qui pourrait permettre le bonheur en faisant vivre les pauvres et les riches dans l'unité, même si ce n'est pas encore le cas dans la réalité: ${ }^{33}$ 'aucune commodité pour les gens de pied; point de trottoirs' $(\mathrm{I}, 62)$.

Que son regard se tourne vers un bouquet de violettes à la Halle; vers les chaussures dont s'occupe le savetier; vers les bons ouvrages qui circulent grâce aux colporteurs; vers les dictionnaires utiles; vers les greniers, ces 'hautes demeures' de l'esprit (I, 30); vers des oranges et des citrons, 'fruits aussi beaux que salubres' (I, 932); vers 'le biscuit de pommes de terre qui l'emporte sur le biscuit de froment' (I, 861-63); vers la 'nouvelle poterie' $(I, 504)$ qui remplace les dangereux vases de terre; vers les nouvelles 'fosses vétérinaires aux quatre coins de la ville' (I, 121); tous ces motifs, dans tous les domaines, sont révélateurs du beau qui recèle les promesses d'harmonie d'un Paris dont l'embellissement s'accroît en permanence. Sa foi profonde en l'humanité rend l'écrivain vigoureux $^{34}$ et $c^{\prime}$ est dans une perspective humaniste qu'il part à la conquête du beau et du bien pour une réconciliation entre l'objet-rue et $1^{\prime}$ homme, se faisant 'prophète' du bonheur. ${ }^{35} \mathrm{Si}$ la rue est bruyante, ne reflète-t-elle pas avant tout l'énergie vitale de l'homme et la recherche d'un plaisir esthétique et sensoriel?:

Aujourd'hui les musiciens sont partout. Des chanteurs et des cantatrices montés sur des tréteaux, chantent dans les cafés des ariettes burlesques, et des airs de l'opéra-comique; on y exécute facilement de bonnes symphonies. Un garçontailleur, en prenant son verre de liqueur, y jouit d'un concert que n'ont point eu soixante rois de France. (I, 1267)

Enfin, même si Mercier avait effectué un bilan négatif des artifices de la mode, c'est grâce au goût pour le beau qu'il peut admirer 'deux rangs 
de jolies femmes [qui] ressemblent à un parterre animé de plusieurs couleurs.... Leur ajustement réunit la légèreté, la décence, la fraîcheur et les grâces' (I, 142-43). ${ }^{36}$

\section{b) Entre modernité et tradition:}

Avec Mercier, la culture française semble avoir enfin intégré le chant de l'orgueil technique dans un monde régi encore comme chez Voltaire par la triade platonicienne du Vrai, du Beau et du Bien. Grâce au progrès, $l^{\prime}$ homme peut sortir de sa condition corrompue et misérable ${ }^{37}$ car ' $c$ 'est $l^{\prime}$ heureux siècle où l'on répand plus d'aisance dans le commerce de la vie, où l'on brillante tous les objets, où l'on imagine chaque jour de nouveaux divertissements pour chasser l'immortel ennui' (I, 637-38).

Cueillir à chaque pas, dans chaque rue, sur chaque trottoir des fleurs de beauté, de gaieté, de bonheur, telle aura été l'ambition et la réussite de Mercier en dépit de toutes les raisons de geindre. Grâce effectivement à sa peinture du Paris des travaux et des jours à la fin du XVIIIè siècle, il laisse l'espoir en une vie rendue meilleure grâce au bien-être matériel et montre que sans la dimension matérielle de l'existence, il n'y a plus de bonheur. Son éloge des artisans qui fabriquent ou qui mettent leur esprit au service de l'objet symbolise l'esthétique et l'esprit nouveaux à l'œuvre dans le Tableau. A l'encontre des moralistes, il est capable de conférer aux outils une valeur hautement humaine comme l'affirmait fièrement un pauvre agonisant victime d'une épidémie de fièvre pourpreuse: 'Les outils que vous voyez me procuraient un pain que je mangeais avec délice et je n'ai jamais été jaloux des tables que j'ai pu entrevoir' (I, 651). Le moribond étonnait par ces propos le 'capucin vénérable' venu l'exhorter à mourir en bon chrétien heureux de quitter un monde où il n'aurait connu que des peines. Il se déclarait en effet 'assez content' de son sort et assurait que s'il reprenait 'la santé' il irait 'au chantier.' Le confesseur ne parvenait plus à effrayer son mourant qui remplaçait le pathos de la mort par la satisfaction d'avoir acquis grâce à sa force de travail le bien-être matériel: plaisir de bouche, santé et restauration de son énergie par le sommeil après les fatigues du jour. Il est vrai que Mercier peint le gourbi vide d'objets: 'Il était seul; une botte de paille lui servait de couverture et d'oreiller; pas un meuble, pas une chaise.' Le lecteur serait tenté de voir dans cette peinture une caractéristique de l'ascétisme chrétien; la mort figurant l'abandon du matériel au profit du spirituel. Mais Mercier lui enlève ce plaisir d'interprétation symbolique car d'une part ce dénuement ne résulte pas d'une morale spiritualiste de dépouillement volontaire mais d'une nécessité socioéconomique: 'Il avait tout vendu dans les premiers jours de sa maladie pour quelques gouttes de bouillon.' D'autre part, l'écrivain repère dans ce vide deux objets essentiels: 'Aux murs noirs et dépouillés pendaient 
seulement une hache et deux scies.' Cette description finit par retrouver une dimension symbolique inverse à la tradition chrétienne et à la morale classique: sur le point de mourir, l'ouvrier chante les pouvoirs de sa force de travail (outils et corps) par opposition à une soumission à la volonté divine, au nom des plaisirs de la vie; les objets sont le gage d'un bonheur véritable pour un homme qui saurait 'faire usage de la vie' (I, 652); loin de les rattacher à une vision 'comique' propre aux auteurs burlesques (Scarron, Sorel, Furetière), il les inclut dans une morale du bonheur. Le drame de l'objet n'est plus avec Mercier de nature morale mais de nature économique. Une visite au Mont de Piété le confirma dans la certitude que les objets signifiaient autre chose que la concupiscence, puisque leur dépôt donne 'une idée non équivoque de la disette extrême où sont plongés le plus grand nombre des habitants' (I, 663). Comme dans le cas du pauvre artisan moribond, les objets prennent sens par leur absence qui renvoie à une injustice et non à un choix moral.

Néanmoins, dans cette avancée incontestable, Mercier ne néglige pas les avertissements traditionnels des moralistes; il limite à des degrés divers le règne de l'objet en rappelant la supériorité de l'esprit sur la matière. ${ }^{38}$

Ce n'est que dans la parole, dans l'écriture, que réside au souverain degré l'imitation des choses. Loin de moi les images matérielles pour exprimer les objets matériels! Il ne faut que des idées intellectuelles pour les reproduire, car la reproduction des objets, l'imitation parfaite, sont en nous: n'allez pas les chercher ailleurs. ${ }^{39}$

Il y a bien avec le Tableau de Paris l'émergence d'une culture matérielle même si elle est encore très marquée par la méfiance traditionnelle, qu'elle soit d'origine stoïcienne ou chrétienne à l'égard de tout ce qui ne dépend pas de l'âme ou de la volonté. Mercier, malgré la tradition spiritualiste qui ne lui est pas étrangère, se tourne vers le monde matériel. C'est pourquoi l'écrivain rend compte de la manière dont ce monde produit des effets néfastes et prolonge le malheur du peuple pour des raisons socio-politiques. ${ }^{40}$ Cependant, malgré cette situation, l'homme parvient de temps en temps à la satisfaction par un environnement matériel plus favorable. Ainsi, conformément au rationalisme classique, Mercier ne perd pas de vue le devoir de beauté, et conformément à la philosophie des Lumières, le droit au bonheur. La critique $q^{\prime}$ 'il ne cesse de développer à l'encontre du faste des grands ${ }^{41}$ indiquerait que l'hypothèse n'est pas dénuée de fondement: une morale et une sociologie conjugueraient leurs analyses pour conduire Mercier vers une conception nuancée et originale des rapports des objets et du bonheur, ni tout à fait matérialiste ni tout à fait spiritualiste. Si origi- 
nalité il y a c'est davantage dans l'expression détendue et galante que lui donne Mercier:

Heureuse nation, qui avez de jolis appartements, de jolis meubles, de jolis bijoux, de jolies productions littéraires, qui prisez avec fureur ces charmantes bagatelles, puissiez-vous prospérer longtemps dans vos jolies idées. (I, 641)

\section{HÉLÈNE CUSSAC \\ Université Blaise Pascal Clermont II}

\section{Notes}

1 Marivaux, Le Paysan parvenu (1735), UGE 10/18, 1965, p. 196.

2 Les références se font à l'édition Mercure de France, dir. J. Cl. Bonnet, 1995, Tomes I et II. Le tome et la numérotation des pages sont indiquées à la suite des citations.

3 Ainsi que le souligne P. Frantz dans son article 'L'usage du peuple': 'Banque documentaire, source inépuisable de renseignements sur la vie au XVIIIè siècle, son œuvre a constitué une source directe pour tous les amateurs d'histoire pittoresque,' Louis-Sébastien Mercier, un hérétique en littérature, sous la dir. de J. Cl. Bonnet, Mercure de France (1995), p. 56.

4 Comme l'affirme P. Frantz: 'Les tenants d'une histoire sociale, qui se donne pour objectif de comprendre les hommes au quotidien, dans leurs gestes et leurs trajets, leurs vêtements et leurs nourritures, leurs goûts, leurs croyances, leurs relations avec leur famille, leurs voisins, leurs clients et leurs compagnons, leurs jeux et leurs joies, ces historiens des 'mentalités' devaient trouver eux aussi en Mercier un témoin irremplaçable,' ibid., p. 56.

5 'Cette description leur [pour les Etrangers] sera d'autant plus utile, [ - - ] qu'ils pourront examiner des choses sur lesquelles ils passeraient sans doute, si l'on ne leur en faisait observer les beautés; mais pour rendre la chose plus facile, l'on a suivi les Quartiers et les Rues, autant qu'il a été possible, afin que dans une même course on pût voir plusieurs belles choses,' 'Avertissement,' Description nouvelle de ce qu'il y a de plus remarquable dans la ville de Paris, seconde édition 'augmentée de plusieurs recherches très curieuses' (1687), chez Nicolas Le Gras, 2 Tomes en un volume, in $12^{\circ}$, (1 ${ }^{\text {ère }}$ éd.: 1684$)$.

6 '... je vais à présent parcourir cette Capitale; je dis parcourir, car mon dessein en composant ces "Essais", n'a pas été d'en donner une description générale, suivie et détaillée, je ne parlerai que des Quartiers et des Rues où il s'est passé quelque fait singulier, intéressant et propre à faire connaître quels ont été, en différents temps, les moeurs et les coutumes de la Nation,' Essais Historiques sur Paris (1776), $5^{\text {ème }}$ éd., 3 Tomes, p. 41.

7 Voir nos index topographique et toponymique des 2 premiers tomes de l'ouvrage dans notre Mémoire de Maîtrise: Voir et Penser la ville dans le 'Tableau de Paris' de L. 
S. Mercier, dir. J. Wagner (Univ. Blaise Pascal, Clermont II, juin 1997), pp. $48-54$ et 114-20.

8 Il est en un sens vrai, comme le dit J. R. Mantion, que 'le tout de la production littéraire de Mercier ne paraît s'agencer, prendre corps et forme, qu'autour d'un œil omniprésent et omnipotent, toujours en éveil,' 'L'œil: Modes d'emploi. Les psychés de L. S. Mercier,' Louis-Sébastien Mercier, un hérétique en littérature, dir. J. Cl. Bonnet, Mercure de France (1995), p. 353.

9 Nous renvoyons ici à la première partie de notre Mémoire de Maîtrise: 'Typologie de l'espace mercérien: un regard au risque du désenchantement?' op. cit., pp. 8-47. Par ailleurs, l'abondance du verbe 'voir,' comme chez son contemporain Rétif de la Bretonne caractérise bien le regard 'sans bornes' de Mercier (particulièrement la $61^{\text {ème }}$ Nuit des Nuits de Paris [Folio Gallimard, 1986], pp. 100-04).

10 Mercier a d'autant plus de mérite de se vouloir moderne que l'avènement de l'esprit moderne ne se fit pas sans réserve avant 1789 , comme le montre J. Wagner dans son article 'La modernité dans la critique littéraire du Journal Encyclopédique entre 1756 et 1786,' Colloque La modernité, Paris IV Sorbonne, 11 janvier 1995, à paraître, R.H.L.F., 1999.

11 Journal de Paris, 10 avril 1778. D'après le Dictionnaire d'un polygraphe, G. Bollème, UGE 1978, p. 106.

12 Le bonheur des gens de lettres, dans Mon bonnet de nuit (1784), T. IV, p. 128.

13 Comme l'affirme J. Cl. Bonnet dans l'introduction de l'ouvrage: 'Regrattier de ce que le temps lui apporte au fil de l'eau, l'écrivain est une sorte d'huissier-priseur qui en fait indéfiniment le détail, mais en ayant troqué le triste uniforme noir pour un habit de couleurs.... Voici donc la littérature conviée, pour la première fois de cette façon, à enregistrer les aspects les plus fugaces et les plus oubliés des choses,' p. lxvi.

14 Sur le retour du peuple en littérature, voir J. Wagner: 'Peuple et pouvoir dans le Journal Encyclopédique à la veille de la Révolution française,' SVEC, 287 (1991): 33-57.

15 Comme l'exprime Steven Kaplan, Le meilleur pain du monde. Les boulangers de Paris au XVIIIè siècle, traduit par P. E. Dauzat (Fayard, 1996), p. 780.

16 Réfléchir sur les lieux qu'il traverse et qui font la trame de la vie ordinaire lui permet de matérialiser les principes de notre connaissance, et ainsi, de mieux comprendre notre relation aux choses, notre médiation aux objets et au monde.

17 Le 'langage' des objets que dévoile Mercier n'est pas sans nous rappeler la perception des inscriptions urbaines chez les surréalistes tels qu'un Breton dans Nadja (1928): nous pensons ici à l'enseigne Bois-Charbons.

18 De même que par Robert Challe en 1713 dès le prologue des Illustres Françaises: '... un cavalier fort bien vêtu, mais dont l'habit, les bottes et le cheval crottés faisaient voir qu'il venait de loin, se trouva arrêté dans un de ces embarras, qui arrivaient tous les jours au bout de la rue de Gesvres. Et malheureusement pour lui les carrosses venant à la file de tous les côtés, il ne pouvait se tourner d'aucun. Un valet qui le suivait était dans la même peine; et tous deux en risque d'être écrasés entre les roues des carrosses, si ils avaient fait le moindre mouvement contraire,' Le livre de poche, 1996, pp. 65-66; ainsi que par un Furetière: '... comme la rue était étroite et le ruisseau large, il couvrit de boue le carrosse, le marquis et la demoiselle,' Le Roman bourgeois (Folio Gallimard, 1981), p. 52. 
19 Mercier relate par ailleurs l'accident que Rousseau rapporte dans sa seconde promenade des Rêveries (GF, 1964, pp. 48-49) et qui faillit lui coûter la vie (I, 109); comme il retrace 'la catastrophe du 18 mai 1770, occasionnée par la foule des voitures qui obstruèrent la rue, unique passage ouvert à l'affluence prodigieuse du peuple qui se portait en foule à la triste illumination des Boulevards,' (I, 108).

20 Sur lesquelles l'écrivain revient à l'intérieur de deux chapitres traitant des latrines publiques (Tomes 7 et 11, Vol. II). Ainsi apprenons-nous que 'les terrasses des Tuileries étaient inabordables par l'infection qui s'en exhalait,' (I, 174).

21 L'emplacement de cimetière des Innocents au centre de la ville font que 'les exhalaisons sépulcrales continuent à empoisonner les fidèles,' (I, 117).

22 Que nous en dit encore Mercier si ce n'est que 'cette épouvantable lie s'achemine lentement le long des rues vers la rivière de Seine, et en infecte les bords, où les porteurs d'eau puisent le matin, dans leurs seaux, l'eau que les insensibles Parisiens sont obligés de boire,' (I, 117).

23 'Le renchérissement du vin, sa criminelle falsification ont forcé l'homme de Paris à recourir à l'eau de vie.... Les femmes de portefaix, qui, à Paris, portent des fardeaux énormes, et travaillent comme des hommes, boivent comme eux cette dangereuse liqueur,' (I, 268).

24 'Ce que Paris renferme en meubles d'or et d'argent, en bijoux, en vaisselle plate, est immense.... Dans les maisons des particuliers, vous voyez des pyramides de vaisselle plate.... nous avons dénaturé nos richesses pour les métamorphoser en meubles.... Ayons toujours des anges, des saints d'argent, des vierges d'argent, et point de papier-monnaie, et bientôt nous nous trouverons pauvres,' (I, 56-58).

25 Sur cette question, voir $\mathrm{Ch}$. Thomas, 'La sphère mouvante des modes,' Louis-Sébastien Mercier, un hérétique en littérature, dir. J. Cl. Bonnet, Mercure de France (1995), pp. 33-53.

26 Voir J. L. Vissière, 'Pollution et nuisances urbaines d'après le Tableau de Paris de S. Mercier,' La Ville au XVIIIè siècle, $9^{\text {ème }}$ Colloque du CAER XVIII, 29 avril-1 ${ }^{\mathrm{er}}$ mai 1973 (A. Colin, 1975).

27 Par conséquent, 'quand un homme qui a un peu de crédit est malade, on répand du fumier devant sa porte, pour rompre le bruit du carrosse,' (I, 108).

28 Ainsi que l'a noté Arlette Farge: 'dans les rues parisiennes du XVIIIè siècle, l'atelier, comme le logement, ouvre grand sur le dehors, aussi résonne-t-il des gestes du travail,' Vivre dans la rue à Paris au XVIIIè siècle, Folio Gallimard, 1992 (particulièrement pages 15-20; 32-33; 118-20).

29 Les Décors et les choses dans le roman français du XVIIIè siècle, SVEC, 297 (1992): 207.

30 On pourrait reconnaître ici l'héritage d'un La Mettrie pour qui la gaieté accompagne toutes les voluptés de la vie et qui se plaît à développer un éloge de la joie (voir son Art de jouir), mais Mercier se montre plus sage que le matérialiste qui rend, au-delà de tous les dieux, hommage avant tout à Vénus. Sur ce développement, voir J. Wagner: 'Rire de joie, rire des Dieux ? La Divinité à l'épreuve des mots de La Mettrie à d'Holbach,' Colloque Rire des Dieux, 12-14 février 1998, (org. D. Bertrand) Univ. Blaise Pascal, Clermont II, Actes à paraître aux Presses Universitaires de Clermont II, coll. Littératures (dir. A. Montandon).

31 Histoire des choses banales. Naissance de la consommation, XVIIè-XIXè siècle (Paris: Fayard, 1997). 
32 Au cœur de ces années de misère, Mercier insiste sur la volupté alimentaire et les ressources dont regorge la ville : 'Allez voir sur le quai de la Volaille, pendue à une large crémaillère: là nagent des chapons au gros sel qui cuisent tous ensemble, et qui se communiquent réciproquement leurs sucs restaurants. A toute heure du jour, vous pouvez pêcher un de ces chapons; un excellent jus l'accompagne et vous le mangerez chez vous tout chaud ou à quatre pas de là, en l'arrosant de vin de Bourgogne.... Chapons gras et huîtres fraîches ne vous manqueront jamais à Paris; vous pourrez commencer votre repas à l'heure que vous voudrez; et ailleurs on ne trouve point pour son argent ni huîtres ni chapons au gros sel,' (I, 1063).

33 'Lorsque je songe qu'une demi-douzaine d'âmes sublimes placées sur les trônes opéreraient le bonheur du monde, je m'étonne que cette combinaison de la nature, n'ait pas encore eu lieu,' Lettre à Thomas du 11 février 1767; cité par G. Bollème, op. cit., p. 106.

34 En plus de l'optimisme dont il a hérité des Lumières, comme il le dit dans ses Entretiens du Palais-Royal: 'Au moment de ma naissance, un billet qui portait santé, liberté, gaieté, $\mathrm{m}$ ' était heureusement échu,' septième entretien, Le guignol (Paris: Buisson, 1786), p. 72.

35 'Qu'est-ce que le bonheur? Le bonheur est l'ouvrage de la raison; c'est le parfait accord de nos désirs et de notre pouvoir. Or, l'homme de lettres, amoureux dès l'enfance, de tout ce qui porte l'empreinte de la pensée et du sentiment, s'éclaire à la lumière de l'une, et s'échauffe à la douce chaleur de l'autre. Il trouve des charmes variés où les autres n'aperçoivent qu'une couleur triste et uniforme,' $L e$ Bonheur des gens de lettres, repris in Mon bonnet de nuit, t. IV, p. 128; cité par G. Bollème, op. cit., p. 105.

36 Et grâce à 'l'ouvrier [qui] donne à la soie toutes les formes possibles; c'est de son goût et de son génie que naissent la variété des dessins, la diversité des couleurs artistement réunies, l'imitation des fleurs naturelles' (I, 735). Comme le dit Ch. Thomas: 'Les métiers de la mode sont pour lui le motif de nombreux tableaux vivants, séduisants, troubles du plaisir' (art. cité, p. 42); 'Admirez la main légère de cette marchande de modes, qui décore sérieusement une poupée' (I, 26). 'Assises dans un comptoir à la file l'une de l'autre, vous les voyez à travers les vitres. Elles arrangent ces pompons, ces colifichets, ces galants trophées que la mode enfante et varie. Vous les regardez librement, et elles vous regardent de même. ces boutiques se trouvent dans toutes les rues. A côté d'un armurier qui n'offre que des cuirasses et des épées, vous ne voyez que des touffes de gaz, des plumes, des rubans, des fleurs et des bonnets de femmes,' (I, 1478).

37 'Les moyens pour le bonheur universel sont déjà indiqués. Il ne s'agit plus que du développement; et de là à la pratique, il n'y a qu'un pas. Voyez si telle idée conçue il y a trente ans, ne se trouve pas réalisée aujourd'hui; et jugez de la force de la raison humaine. Quand le génie lui a prêté le tonnerre de sa voix majestueuse, quel peuple, tôt ou tard, ne l'entend point, ne se réveille point de la léthargie où il sommeillait,' De la littérature et des littérateurs, p. 12; cité par G. Bollème, op. cit., p. 107.

38 Mercier réconcilie la philosophie avec le quotidien tâchant bien 'de mettre en harmonie les besoins de la vie avec les préoccupations de la raison studieuse' ainsi que le proposera Karl Gottlob Schelle dans son Art de se promener à la fin du XVIIIè siècle: 'La philosophie se cantonne encore trop souvent dans les sphères de 
la spéculation; rares sont les penseurs qui se hasardent à appréhender les objets de la vie,' cité par D. Roche, op. cit., introduction, p. 9.

39 Journal de Paris, 10 fructidor An V.

40 Mercier ne supporte pas le mépris des grands pour la vie des petits: 'Le peuple semble un corps séparé des autres ordres de l'Etat; les riches et les grands qui ont un équipage, ont le droit barbare de l'écraser et de le mutiler dans les rues,' (I, 62).

41 Tout particulièrement du fait de leur insensibilité: 'L'indifférence pour ces sortes d'accidents fait voir que l'on croit que tout doit servir le faste des grands,' (I, 62). 